\title{
AN INEQUALITY FOR SYMPLECTIC FILLINGS OF THE LINK OF A HYPERSURFACE $K 3$ SINGULARITY
}

\author{
HIROSHI OHTA \\ Graduate School of Mathematics, Nagoya University \\ Nagoya, 464-8602, Japan \\ E-mail: ohta@math.nagoya-u.ac.jp \\ KAORU ONO \\ Department of Mathematics, Hokkaido University \\ Sapporo, 060-0810, Japan \\ E-mail:ono@math.sci.hokudai.ac.jp
}

\begin{abstract}
Some relations between normal complex surface singularities and symplectic fillings of the links of the singularities are discussed. For a certain class of singularities of general type, which are called hypersurface $K 3$ singularities in this paper, an inequality for numerical invariants of any minimal symplectic fillings of the links of the singularities is derived. This inequality can be regarded as a symplectic/contact analog of the 11/8-conjecture in 4-dimensional topology.
\end{abstract}

1. Introduction. The geometry of the link $L$ of an isolated singularity $O$ of a complex algebraic surface $V$ is deeply related to properties of the singularity. For example, Mumford's theorem says that if $L$ is a 3-sphere, then $O$ is actually a non-singular point. The link $L$ carries a natural contact structure given by the maximal complex tangency $\xi$. (See Section 2 for the precise definition.) We study symplectic fillings of the contact manifold $(L, \xi)$. The typical examples of symplectic fillings of the link of a singularity are resolutions of the singularity and a smoothing (Milnor fiber), if it exists. In this note, we discuss some relations between numerical invariants of the singularity and properties of the set of symplectic fillings of the link.

In the series of papers [OO1],[OO2],[OO3], we considered the classes of simple singularities and simple elliptic singularities, and completely classified the symplectic deformation

2000 Mathematics Subject Classification: Primary 57R17; Secondary 32S25, 53D05.

Key words and phrases: symplectic fillings, isolated surface singularities of general type, Arnold's 14 exceptional unimodular singularities, $K 3$ surfaces.

The paper is in final form and no version of it will be published elsewhere. 
types (in particular, diffeomorphism types) of minimal symplectic fillings of their links. However, we cannot expect to determine all the deformation types of minimal symplectic fillings of the link of a singularity, in general. In fact, in [OO4] we gave some examples of isolated singularities whose links have infinitely (countably) many distinct minimal symplectic fillings. From the point of view of the classification theory of normal surface singularities explained in Section 3, these examples belong to the class of singularities of general type. In this note, we will study general properties of minimal simplectic fillings of the links of certain normal surface singularities of general type.

2. Preliminaries. In this section we briefly recall some basic notions and facts about symplectic fillings which we will use.

Let $L$ be the link of an isolated singularity in a complex algebraic surface $V$. $L$ carries a natural contact structure $\xi$ defined by $\xi=T L \cap J T L$. Here $J$ is a complex structure on $V$. Note that the contact structure on a $(4 k+3)$-dimensional manifold induces a natural orientation on it. In particular, $L$, which is 3-dimensional, is naturally oriented. A compact symplectic manifold $(W, \omega)$ is called a strong symplectic filling (resp. strong concave filling) of the contact manifold $(L, \xi)$ if the orientation of $L$ as a contact manifold is the same as (resp. opposite to) its orientation as the boundary of the symplectic manifold $W$ and there exists a 1-form $\lambda$ on $L$ such that $\xi=\operatorname{ker} \lambda$ and $d \lambda=\left.\omega\right|_{L}$. This condition is equivalent to the existence of an outward (resp. inward) normal vector field $X$ around $\partial W$ such that $\mathcal{L}_{X} \omega=\omega$ and $i(X) \omega$ vanishes on $\xi$. Hereafter, we call strong symplectic fillings simply 'symplectic fillings'. (Recall that when $L$ is a rational homology 3 -sphere, the notion of strong symplectic filling is equivalent to the notion of weak symplectic filling, see Lemma 1.1 in [OO1]. Here 'weak symplectic fillings' means that we replace the condition $d \lambda=\left.\omega\right|_{L}$ by $\left.\omega\right|_{\xi}>0$. We do not use this fact in this note.) This condition may be regarded as a symplectic analog of (pseudo) convexity for the boundary. Such a boundary (or a hypersurface) is said to be of contact type. Simple examples are the boundaries of convex domains, or more generally star-shaped domains in a symplectic vector space. Namely, if the convex domain contains the origin, the Euler vector field $\sum\left(x_{i} \frac{\partial}{\partial x_{i}}+y_{i} \frac{\partial}{\partial y_{i}}\right)$ is a desired outward vector field. Here $\left\{x_{i}, y_{i}\right\}$ are the canonical coordinates.

A symplectic 4-manifold is called minimal if it does not contain any symplectically embedded spheres of self intersection number -1 (such a sphere would be referred to as a symplectic (-1)-curve).

Two symplectic structures $\omega_{0}$ and $\omega_{1}$ on $X$ are called symplectic deformation equivalent if there exists a path of symplectic structures $\omega_{t}$ joining $\omega_{0}$ and $\omega_{1}$.

3. Normal singularities vs symplectic fillings. Let $O$ be a normal surface singularity and $(L, \xi)$ its link with the contact structure $\xi$ defined in Section 2. As we mentioned above, our working hypothesis is that numerical invariants of the singularity $O$ deeply reflect properties of the set of minimal symplectic fillings of the link $(L, \xi)$ of $O$. To see this correspondence, we first explain the singularity side. There is an attempt to build a classification theory of normal surface singularities modeled on Kodaira-Enriques classification theory of complex surfaces. Following [I1], we review the classification theory of singularities very briefly. Let $(X, x)$ be a normal isolated singularity (not necessary 
surface singularity) in $X$, and $n=\operatorname{dim}_{\mathbf{C}} X$. We define the Kodaira dimension of $(X, x)$, which we denote by $\kappa_{\delta}$, as follows. Firstly, we define

$$
\delta_{m}(X, x):=\operatorname{dim}_{\mathbf{C}} \Gamma\left(\widetilde{X} \backslash E, \mathcal{O}_{\widetilde{X}}\left(m K_{\tilde{X}}\right)\right) / \Gamma\left(\widetilde{X}, \mathcal{O}_{\widetilde{X}}\left(m K_{\widetilde{X}}+(m-1) E\right)\right)
$$

for any natural number $m$. Here $f: \widetilde{X} \rightarrow X$ is a good resolution of singularity, $E$ is the exceptional divisor and $K_{\widetilde{X}}$ is the canonical divisor of $\widetilde{X}$. Originally, $\delta_{m}(X, x)$ was defined in $[\mathrm{W}]$ by

$$
\delta_{m}(X, x):=\operatorname{dim}_{\mathbf{C}} \Gamma\left(\tilde{X} \backslash E, \mathcal{O}_{\tilde{X}}\left(m K_{\tilde{X}}\right)\right) / L^{2 / m}(X \backslash\{x\}),
$$

where the denominator denotes the set of $L^{2 / m}$-integrable $m$-ple holomorphic $n$-forms on $X \backslash\{x\}$; according to $[\mathrm{Sak}]$, these two definitions coincide. One can show that $\delta_{m}(X, x)$ is independent of the choice of the good resolution, and that $\delta_{m}(X, x)<\infty$. This gives a version of plurigenera. Then Ishii's result [I2] implies that for any normal isolated singularity, we have either

(i) $\delta_{m}(X, x)=0$ for all $m$, or

(ii) for some $k=0,1,2, \ldots, n-2, n=\operatorname{dim} X$,

$$
0<\limsup _{m \rightarrow \infty} \frac{\delta_{m}(X, x)}{m^{k}}<\infty .
$$

When (i) holds, we define $\kappa_{\delta}(X, x)=-\infty$. When (ii) holds, we define $\kappa_{\delta}(X, x)=k$.

Here is the classification table of normal Gorenstein surface singularities by Kodaira dimension. (We omit the classification table for the non-Gorenstein case here; it can be obtained by taking quotients of Gorenstein singularities.)

Table 3.1

\begin{tabular}{|c|c|}
\hline$\kappa_{\delta}$ & Gorenstein surface singularity \\
\hline$-\infty$ & simple singularity \\
\hline 0 & simple elliptic singularity, cusp singularity \\
\hline 2 & other (of general type) \\
\hline
\end{tabular}

We omit precise definitions of each class of singularities in the table. The interested reader may consult [Sa2], [I1], for example.

On the other hand, we have the following table on symplectic deformation types of minimal symplectic fillings of the links of singularities.

Table 3.2

\begin{tabular}{|c|c|}
\hline$\kappa_{\delta}$ & Symplectic deformation types of minimal symplectic fillings \\
\hline$-\infty$ & \multicolumn{1}{|c|}{ unique [OO3] } \\
\hline 0 & $\begin{array}{l}\text { simple elliptic singularity : } \\
\text { (smoothing or minimal resolution, completely classified [OO2]). } \\
\text { cusp singularity : (?) }\end{array}$ \\
\hline 2 & $? ?$ \\
\hline
\end{tabular}


For other related (non-Gorenstein and $\kappa_{\delta}=-\infty$ cases) results, see [Mc], [BO], [L]. From these two tables, we can see that when $\kappa_{\delta}=-\infty$, the set of minimal symplectic fillings can be viewed as 'rigid', and when $\kappa_{\delta}=0$, it has a 'semi-rigid' aspect. (For the cusp singularity case, we do not know the complete list of minimal symplectic fillings.) When $\kappa_{\delta}=2$, which is the case of the singularity of general type, we do not know how big (or small) the set of minimal symplectic fillings of the link is, in general. We note that in [OO4] we gave some examples of singularities whose links have infinitely many distinct minimal symplectic fillings. The examples of singularities in [OO4] are of general type, hence in general we cannot expect to get a complete list of minimal symplectic fillings of a link of singularity of general type. One possible way to approach this problem is to study 'sociology' of symplectic fillings. For example, it would be interesting to find some general inequality for minimal symplectic fillings of the link of singularity of general type, like the Noether inequality or the Miyaoka-Yau inequality for minimal surfaces of general type. In the next section, we give an inequality which is, in spirit, along this line of research.

4. An inequality. In this section we consider the class of singularities of Brieskorn type. Let $f \in \mathbf{C}[x, y, z]$ be a polynomial in three variables. For integers $p, q, r \geq 2$, we let

$$
f_{p, q, r}(x, y, z)=x^{p}+y^{q}+z^{r} .
$$

Consider the hypersurface $f_{p, q, r}^{-1}(0)$ in $\mathbf{C}^{3}$. The origin $O$ is an isolated singularity of $f_{p, q, r}^{-1}(0)$. We call $(p, q, r)$ the type of singularity, and denote by $\Sigma(p, q, r)$ its link.

LEMMA 4.1. (1) If $\frac{1}{p}+\frac{1}{q}+\frac{1}{r}>1$, then $\kappa_{\delta}=-\infty$.

(2) If $\frac{1}{p}+\frac{1}{q}+\frac{1}{r}=1$, then $\kappa_{\delta}=0$.

(3) If $\frac{1}{p}+\frac{1}{q}+\frac{1}{r}<1$, then $\kappa_{\delta}=2$.

Proof. (1) This is the case of simple singularities. (2) The values $(p, q, r)=(2,3,6)$, $(2,4,4)$ and $(3,3,3)$ correspond to the simple elliptic singularities of types $\widetilde{E}_{8}, \widetilde{E}_{7}, \widetilde{E}_{6}$, respectively, see for instance [Sa2]. (3) The minimal resolution diagram for the singularity defined by $f_{p, q, r}$ is contractible. See for instance [OW]. A cusp singularity does not appear in this case. Thus $\frac{1}{p}+\frac{1}{q}+\frac{1}{r}<1$ implies $\kappa_{\delta}=2$ using Table 3.1.

Next we would like to consider singularities of general type. We start with the simplest example of case (3) in Lemma 4.1, namely, $(p, q, r)=(2,3,7)$.

THEOREM 4.2. Let $X$ be any minimal symplectic filling of the link of the singularity of type $(2,3,7)$. Assume that $b_{2}^{+}(X) \neq 0$. Then

$$
3 \operatorname{sign}(X)+2 e(X) \geq 3 \operatorname{sign}(V(2,3,7))+2 e(V(2,3,7))=2 .
$$

Here $V(2,3,7)$ is the Milnor fiber defined by

$$
V(2,3,7)=\left\{(x, y, z) \in \mathbf{C}^{3} \mid x^{2}+y^{3}+z^{7}=1\right\} .
$$

REMARK 4.3. The minimal resolution of singularity is an example of minimal symplectic filling with negative definite intersection form. In this case, the inequality obviously does not hold. This shows that the assumption $b_{2}^{+}(X) \neq 0$ is really necessary.

Proof of Theorem 4.2. We consider the Milnor fiber $V(2,3,7)$. According to Pinkham $[\mathrm{P}]$, $V(2,3,7)$ can be compactified into a $K 3$ surface $W$ by using an appropriate embedding of 
the Milnor fiber $V(2,3,7)$ into a certain weighted projective space, see also [Sa1], [Sa2]. We let

$$
W=V(2,3,7) \cup_{\Sigma(2,3,7)} V(2,3,7)^{\text {out }} .
$$

Recall that the intersection matrix of $V(2,3,7)$ is given by $E_{8} \oplus 2\left(\begin{array}{ll}0 & 1 \\ 1 & 0\end{array}\right)$. (Here $E_{8}$ is the negative definite Cartan matrix of type $E_{8}$.) This follows from the fact that $b_{2}(V(2,3,7))=12, \operatorname{Sign}(V(2,3,7))=-8$ and $c_{1}(V(2,3,7))=0$. See [Mi] and [B] for this calculation. Thus the intersection form of $V(2,3,7)^{\text {out }}$ is equivalent to $E_{8} \oplus\left(\begin{array}{ll}0 & 1 \\ 1 & 0\end{array}\right)$. (We may also deduce this by using explicit description $[\mathrm{P}]$, [Sa1] of the compactifying divisor.) Note that $V(2,3,7)^{\text {out }}$ gives a concave filling of the link $\Sigma(2,3,7)$.

Now let $X$ be any minimal symplectic filling of the link $\Sigma(2,3,7)$. Then we can glue $X$ and $V(2,3,7)^{\text {out }}$ together to obtain a closed smooth symplectic 4 -manifold $\left(Z, \omega_{Z}\right)$ :

$$
Z=X \cup_{\Sigma(2,3,7)} V(2,3,7)^{\text {out }} .
$$

See Lemma 2.1 in [OO1] for the argument on gluing symplectic structures. We call $Z$ a compactification of the filling $X$.

We denote by $K_{Z}$ the canonical bundle of $Z$. Assume that $c_{1}\left(K_{Z}\right)=0$. Since $c_{1}^{2}\left(K_{Z}\right)=$ $3 \operatorname{Sign}(Z)+2 e(Z)$, the Novikov additivity and the equality $e(Z)=e(X)+e\left(V(2,3,7)^{\text {out }}\right)$ imply that $3 \operatorname{Sign}(X)+2 e(X)=2$. Therefore the equality holds in this case. From now on, we will assume that $c_{1}\left(K_{Z}\right) \neq 0$. Let $\lambda$ be a contact form of $\xi$ on $\Sigma(2,3,7)$ such that $\left.\omega_{Z}\right|_{\Sigma(2,3,7)}=d \lambda$. Denote by $R_{\lambda}$ the Reeb vector field for $\lambda$. Namely, $i\left(R_{\lambda}\right) \omega_{Z}=0$, $i\left(R_{\lambda}\right) \lambda=1$. We can take a compatible almost complex structure $J$ on $Z$ such that $J$ is compatible with $\lambda$, i.e., $J$ preserves $\xi$ and $J$ sends $R_{\lambda}$ to an inward normal vector field for the symplectic filling $X$.

Proposition 4.4. The compactification $Z$ is minimal.

Proof. Suppose $Z$ is not minimal. Then there exists an embedded symplectic $(-1)$ curve $E$. Since $b_{2}^{+}(X) \geq 1$ and $b_{2}^{+}\left(V(2,3,7)^{\text {out }}\right)=1$, we have $b_{2}^{+}(Z) \geq 2$. Then Taubes' theorem [T1] tells us that the Poincaré dual of $c_{1}\left(K_{Z}\right)$ can be represented by a pseudo-holomorphic curve, which we denote by $D$. If $E$ is not contained in $D$, we have $E \cdot D \geq 0$ because of the positivity of intersection of pseudo-holomorphic curves. But this contradicts the adjunction formula. Therefore $E$ is contained in $D$. On the other hand, we have the following result:

Lemma 4.5. $D \subset X$.

With Lemma 4.5 in place, we see that $E \subset X$, which contradicts the minimality of $X$. This proves that $Z$ is minimal. Therefore, it is enough to prove Lemma 4.5 to prove Proposition 4.4. We assume that $D \cap V(2,3,7)^{\text {out }} \neq \emptyset$. Since $D$ is a symplectic curve, we have

$$
\int_{D \cap V(2,3,7)^{\text {out }}} \omega_{Z}>0 .
$$

Note that $\left.K_{Z}\right|_{V(2,3,7)^{\text {out }}}=\left.K_{W}\right|_{V(2,3,7)^{\text {out }}}$ is trivial, because $W$ is a $K 3$ surface. Hence $D \cap V(2,3,7)^{\text {out }}$ is homologous to zero in $V(2,3,7)^{\text {out }}$ relative to $\partial V(2,3,7)^{\text {out }}$. Thus $D \cap V(2,3,7)^{\text {out }}$ is homologous to a relative 2 -cycle $D^{\prime}$ in $\partial V(2,3,7)^{\text {out }}$ with boundary 
$\partial\left(D \cap V(2,3,7)^{\text {out }}\right)$. By Stokes' theorem, we have

$$
\int_{D \cap V(2,3,7)^{\text {out }}} \omega_{Z}=\int_{D^{\prime}} d \lambda=\int_{\partial\left(D \cap V(2,3,7)^{\text {out }}\right)} \lambda .
$$

Recall that $\left.\omega_{Z}\right|_{\Sigma(2,3,7)}=d \lambda$ and $\lambda$ is a contact form of $\xi$ so that $\operatorname{ker} \lambda=\xi$. Since $V(2,3,7)^{\text {out }}$ is a concave filling and we choose $J$ compatible with $\lambda$, the right hand side of the above equality is negative. This argument is used in the proof of Lemma 3.2 in [OO1]. This leads to a contradiction. We have finished the proof of Lemma 4.5 and also the proof of Proposition 4.4.

Using Taubes's theorem [T2], we get $c_{1}^{2}\left(K_{Z}\right) \geq 0$. The equality $c_{1}^{2}\left(K_{Z}\right)=3 \operatorname{Sign}(Z)+$ $2 e(Z)$ together with the Novikov additivity and the equality $e(Z)=e(X)+e\left(V(2,3,7)^{\text {out }}\right)$ leads to

$$
\begin{aligned}
3 \operatorname{sign}(X)+2 e(X) & \geq-\left(3 \operatorname{sign}\left(V(2,3,7)^{\text {out }}\right)+2 e\left(V(2,3,7)^{\text {out }}\right)\right) \\
& =3 \operatorname{sign}(V(2,3,7))+2 e(V(2,3,7))=2,
\end{aligned}
$$

which proves Theorem 4.2.

5. Hypersurface $K 3$ singularities. Theorem 4.2 can be proved for a more general class of singularities. The above proof is based on the following two observations:

(5.1) The Milnor fiber (or smoothing) can be compactified (by a compactifying divisor) into a $K 3$ surface (or more generally, a projective surface with $K=0$ ).

(5.2) The intersection form of a regular neighborhood of the compactifying divisor has at least one positive eigenvalue.

The singularity of type $(2,3,7)$ which we dealt with in the previous section is one of Arnold's 14 exceptional unimodular singularities [A]. Pinkham [P] actually showed that the these singularities have the properties (5.1) and (5.2). Moreover, K. Saito [Sa1] obtained a complete list of singularities defined by a weighted homogeneous polynomial in three variables whose Milnor fiber compactifies into a $K 3$ surface; there are 49 such singularities. See the part of Table 2 in [Sa1] with $\epsilon<0$. In this note we call them hypersurface K3 singularities. They are Gorenstein singularities of general type in the sense of Section 3. All singularities on Saito's list have the properties (5.1) and (5.2). In fact, we find that $b_{2}^{+}=1$ for the regular neighborhood of the compactifying divisor. This follows from the result of M. Saito $[\mathrm{MoS}]$ that $b_{2}^{+}$of the corresponding Milnor fiber of the singularity equals twice the number of negative exponents in Table 1 in [Sa1]. (As a result, we find that $b_{2}^{+}$of the Milnor fiber is 2 for any hypersurface $K 3$ singularity. See also the formula (5.7.4) in [Sa1].) Therefore, for the class of hypersurface $K 3$ singularities, we obtain the following.

THEOREM 5.3. Let $X$ be any minimal symplectic filling of the link of a hypersurface K3 singularity. Assume that $b_{2}^{+}(X) \neq 0$. Then

$$
3 \operatorname{sign}(X)+2 e(X) \geq 3 \operatorname{sign}(V)+2 e(V) .
$$

Here $V$ is the Milnor fiber of the singularity. 
REMARK 5.4. (1) We assume $b_{1}(X)=0$. Then the inequality is equivalent to $\operatorname{sign}(X) \geq$ $-\frac{2}{3} b_{2}(X)+\frac{1}{3}(12-\mu)$, where $\mu=b_{2}(V)$ is the Milnor number of the singularity. We consider the case of $\operatorname{sign}(X)<0$. Then the inequality is equivalent to

$$
b_{2}(X) \geq \frac{12}{8}|\operatorname{sign} X|+\frac{1}{2}(12-\mu) .
$$

Here $10 \leq \mu \leq 21$ for hypersurface $K 3$ singularities, see [Sa1]. This inequality can be regarded as a relative analog (in symplectic/contact geometry) of the famous 11/8conjecture which says that

$$
b_{2}(X) \geq \frac{11}{8}|\operatorname{sign} X|
$$

for any closed smooth spin 4-manifold $X$. As for the $11 / 8$-conjecture, Furuta $[\mathrm{F}]$ proved that

$$
b_{2}(X) \geq \frac{10}{8}|\operatorname{sign} X|+2 .
$$

(2) Here we consider a class of hypersurface singularities. However, it is not necessary to assume that the singularity is hypersurface or Gorenstein for the proof to go through. In fact, we can generalize the above theorem to singularities having properties (5.1) and (5.2). On the right hand side of the resulting inequality, $V$ will stand for any smoothing of the singularity.

Acknowledgements. The authors would like to thank Kyoji Saito and Atsushi Takahashi for helpful information relating to the hypersurface $K 3$ singularities.

Research of the first author was partially supported by Grant-in-Aid for Scientific Research No. 19340017, JSPS, and research of the second author was partially supported by Grant-in-Aid for Scientific Research Nos. 17654009 and 18340014, JSPS.

Added in proof. The terminology "hypersurface simple $K 3$ singularity" has appeared in T. Yonemura, On hypersurface simple K3 singularities, Tohoku Math. J. 42 (1990), 351-380. They are isolated singularities in dimension 3 . Hypersurface $K 3$-singularities in this paper are isolated singularities in dimension 2 .

\section{References}

[A] V. I. Arnold, Critical points of smooth functions and their normal forms, Russian Math. Surveys 30 (1975) no. 5, 1-75.

[BO] M. Bhupal and K. Ono, Symplectic fillings of quotient surface singularities, preprint, 2003.

[B] E. Brieskorn, Beispiele zur Differentialtopologie von Singularitäten, Invent. Math. 2 (1966) 1-14.

[F] M. Furuta, Monopole equation and $\frac{11}{8}$ conjecture, Math. Res. Lett. 8 (2001), 279-291.

[I1] S. Ishii, Introduction to Singularity Theory, Springer-Verlag, Tokyo, 1997 (in Japanese).

[I2] S. Ishii, The asymptotic behavior of pluri-genera for a normal isolated singularity, Math. Ann. 286 (1990) 803-812.

[L] P. Lisca, On lens spaces and their symplectic fillings, Trans. Amer. Math. Soc. 360 (2008), 765-799. 
[Mc] D. McDuff, The structure of rational and ruled symplectic 4-manifolds, J. Amer. Math. Soc. 3 (1990), 679-712.

[Mi] J. F. Milnor, Singular Points of Complex Hypersurfaces, Princeton Univ. Press, 1968.

[OO1] H. Ohta and K. Ono, Simple singularities and topology of symplectically filling 4manifolds, Comm. Math. Helv. 74 (1999), 575-590.

[OO2] H. Ohta and K. Ono, Symplectic filling of the link of simple elliptic singularities, J. Reine Angew. Math. 565 (2003), 183-205.

[OO3] H. Ohta and K. Ono, Simple singularities and symplectic fillings, J. Differential Geom. 69 (2005), 1-42.

[OO4] H. Ohta and K. Ono, Examples of isolated surface singularities whose links have infinitely many symplectic fillings, J. Fixed Point Theory Appl. 3 (2008), 51-56.

[OW] P. Orlik and P. Wagreich, Isolated singularities of algebraic surfaces with $\mathbf{C}^{*}$ action, Ann. of Math. 93 (1971), 205-228.

[P] H. Pinkham, Singularités exceptionnelles, la dualité étrange d'Arnold et les surfaces K3, C. R. Acad. Sci. Paris Sér. A 284 (1977) 615-618.

[Sa1] K. Saito, Algebraic surfaces for regular systems of weights, in: Algebraic Geometry and Commutative Algebra, Vol. II, Kinokuniya, Tokyo, 1988, 517-614.

[Sa2] K. Saito, Around the theory of the generalized weight system: Relations with singularity theory, the generalized Weyl group and its invariant theory, etc., Amer. Math. Soc. Transl. (2) 183 (1998), 101-143.

[MoS] M. Saito, On the exponents and the geometric genus of an isolated hypersurface singularity, in: Proc. Symposia Pure Math. 40, Amer. Math. Soc., 1983, 465-472.

[Sak] F. Sakai, Kodaira dimension of complements of divisors, in: Complex Analysis and Algebraic Geometry, a collection of papers dedicated to K. Kodaira, Iwanami-Cambridge, 1977, 139-157.

[T1] C. H. Taubes, The Seiberg-Witten invariants and symplectic forms, Math. Res. Lett. 1 (1994), 809-822.

[T2] C. H. Taubes, The Seiberg-Witten and the Gromov invariants, Math. Res. Lett. 2 (1995), 221-238.

[W] K. Watanabe, On plurigenera of normal isolated singularities I, Math. Ann. 250 (1980) 65-94. 